\title{
Serum lubricin levels in patients with juvenile idiopathic arthritis
}

\author{
Rabia Miray Kisla Ekinci ${ }^{1}$ ID , Sibel Balci $^{1}$ ID , Fatma Coban² ID $^{2}$, Atil Bisgin² ID \\ ${ }^{1}$ Department of Pediatric Rheumatology, Cukurova University Faculty of Medicine, Adana, Turkey \\ ${ }^{2}$ Department of Medical Genetics, Cukurova University Faculty of Medicine, Adana, Turkey
}

\begin{abstract}
Objectives: Lubricin, encoded by the proteoglycan 4 (PRG4) gene, is mainly responsible for lubricating joints. However, there is expanding evidence on its involvement in inflammatory pathways. Juvenile idiopathic arthritis (JIA) is a heterogeneous group of chronic arthritides with an unknown origin in children aged below 16 years. It is characterized by chronic joint inflammation, including synovial inflammation, and may result in cartilage destruction. We aimed to determine whether serum lubricin levels are affected in JIA patients.

Material and methods: This cross-sectional study included children diagnosed with JIA and 28 healthy controls. The patients were divided into two groups according to the presence of remission at the time of study. Lubricin protein analysis was performed by the enzyme-linked immunosorbent assay method. Serum samples were obtained at the study enrollment, and lubricin levels were measured once, and compared between JIA patients and healthy controls, and between JIA patients with active disease and remission.

Results: The study included 52 JIA patients ( 28 female, 24 male) and 28 healthy controls (18 female, 10 males). The mean age at study enrollment was $11.66 \pm 4.41$ years and $12.72 \pm 4.52$ years in the IIA patient and control groups, respectively. Although median serum lubricin level did not differ between JIA patients (median: $0.66 \mathrm{ng} / \mu \mathrm{l}$, range: $0.02-3.85 \mathrm{ng} / \mu \mathrm{l}$ ) and healthy controls (median: $0.52 \mathrm{ng} / \mu \mathrm{l}$, range: $0.06-3.84 \mathrm{ng} / \mu \mathrm{l})$, it was statistically significantly higher in patients with active disease (median: $1.58 \mathrm{ng} / \mu$, range: $0.08-3.85 \mathrm{ng} / \mu \mathrm{l}$ ) than both patients in remission (median: $0.57 \mathrm{ng} / \mu \mathrm{l}$, range: $0.02-3.57 \mathrm{ng} / \mu \mathrm{l}$ ) and healthy controls. A low degree positive correlation was also found between serum lubricin levels and erythroid sedimentation rate of the JIA patients $(r=0.383$ and $p=0.011$.

Conclusions: This is the first study investigating serum lubricin levels in JIA patients, and we found elevated serum lubricin levels in IIA patients with active disease. Further studies are needed to clarify our results.
\end{abstract}

Key words: juvenile idiopathic arthritis, lubricin, proteoglycan 4.

\section{Introduction}

Lubricin, encoded by the proteoglycan 4 (PRG4) gene, is a mucin-like molecule and includes proline, serine and threonine that provides a scaffold for glycosylation, high viscosity and low friction [1, 2]. It is suggested to have protective effects against synovial hyperplasia and cartilage deterioration in the joint spaces.

Experimental models of osteoarthritis showed that lubricin retards cartilage degeneration, enhances carti- lage repair and reduces chondrocyte apoptosis [3]. Lubricin levels were also diminished in synovial fluid of inflammatory arthritis [4]. Besides the lubricating role, it was previously proposed that the sulfated oligosaccharide epitopes on lubricin could be part of the L-selectin ligand, responsible for recruitment of leucocytes to inflammatory sites [5].

Furthermore, lubricin was recently suggested to act as an antagonist for Toll like receptor (TLR) 2 and TLR4, preventing its activation in inflammatory arthritis [6].

Address for correspondence:

Rabia Miray Kisla Ekinci, University of Health Sciences, Adana City Training and Research Hospital, Yuregir, 01060, Adana, Turkey, e-mail: mir kisla@hotmail.com

Submitted: 04.05.2021; Accepted: 22.11.2021 
Moreover, a recent study proposed a new mechanism of lubricin, including inhibition of interleukin $1 \beta$ (IL-1 $\beta$ ) and tumor necrosis factor- $\alpha$ (TNF- $\alpha$ ) via CD44 binding [7].

Juvenile idiopathic arthritis (JIA) is a heterogeneous group of chronic arthritides with an unknown origin in children aged below 16 years. It is characterized by chronic inflammation of joints, including synovial inflammation, and may result in cartilage destruction $[8,9]$. A heterogeneous genetic susceptibility, environmental triggers, including infections and trauma, and alterations in immunologic self-tolerance were the main pathogenetic mechanism in JIA [9].

The management of JIA includes pharmacological intervention, physical/occupational therapy, and psychosocial support. With the improvements in anti-inflammatory medications, particularly biological agents, prevention of the joint from cartilage destruction and relevant damage became the major aim of JIA treatment [9].

In the view of the recently proposed anti-inflammatory effects of lubricin, we aimed to investigate serum lubricin levels in JIA patients according to the presence of active disease or remission.

\section{Materials and methods \\ Patient selection}

This cross-sectional study included JIA patients with at least 12-month follow-up duration. Patients were enrolled in the study between July 2019 and November 2019. All patients were classified as having JIA according to the International League of Associations for Rheumatology criteria [10]. The control group consisted of 28 healthy children aged $6-18$ years, and admitted to the pediatrics outpatient clinic for routine follow-up visits.

The local ethics committee of our medical school approved the present study (date: 14 June 2019, number: 89/6).

\section{Data collection}

The demographic features including age and gender and JIA subtypes were retrospectively collected from the medical files of the patients. Erythrocyte sedimentation rate (ESR) and C-reactive protein (CRP) were obtained

Table I. Demographic characteristics of patients with juvenile idiopathic arthritis and healthy controls

\begin{tabular}{lccc|}
\hline Parameters & $\begin{array}{c}\text { JIA } \\
(n=52)\end{array}$ & $\begin{array}{c}\text { Control group } \\
(n=28)\end{array}$ & $p$ \\
\hline Gender (female) $[n(\%)]$ & $28(54.9)$ & $18(64.3)$ & 0.419 \\
\hline Age (mean \pm SD) & $11.66 \pm 4.41$ & $12.72 \pm 4.52$ & 0.328 \\
\hline
\end{tabular} $\begin{aligned} & \text { JIA - juvenile idiopathic arthritis, SD - standard deviation, } p \text {-values } \\
& \text { below 0.05 are significant. }\end{aligned}$

at the study enrollment. The patients were separated into two groups according to the presence of remission at the time of study, based on the American College of Rheumatology provisional criteria for clinically inactive disease [11].

Serum samples were obtained at the study enrollment, and serum lubricin levels were measured once. The lubricin levels were compared between JIA patients and healthy controls, and also between JIA patients with active disease and remission.

\section{Serum lubricin analysis}

Serum was collected from the blood samples taken from the patients, and centrifugated at $2500 \mathrm{rpm}$ for 5 minutes. The enzyme-linked immunosorbent assay (ELISA) method and a PRG4/Lubricin ELISA kit (USCN, Wuhan) were used for protein analysis. ELISA plates were read by BIOTEK 800 TS.

\section{Statistical analysis}

Statistical tests were performed by SPSS 20.0 statistical software (IBM SPSS Statistics). Categorical variables were indicated as numbers and percentages. Continuous variables were summarized as mean and standard deviation or median and minimum-maximum after their distributions were checked. The $\chi^{2}$ test and Student's t-test were utilized for comparison of two categorical and parametric continuous variables, respectively. Also, non-parametric continuous variables were compared between three groups with the Kruskal-Wallis test. The significance level of the $p$-value was considered to be 0.05 .

\section{Results}

The study included 52 JIA patients and 28 healthy controls. The demographic features were statistically similar between patients and the control group (Table I). Of these JIA patients, 34 (65.4\%) had active disease and 18 (34.6\%) were in off-treatment remission. Of 34 patients with active disease, 14 were receiving only methotrexate, and 20 patients were receiving both methotrexate and a biological disease modifying antirheumatic drug at the time of the study. Nineteen patients (38.5\%) had oligoarticular JIA, 10 (19.2\%) had polyarticular JIA, 7 (13.5\%) had systemic JIA, 13 (25\%) had enthesitis-related arthritis and 3 (5.8\%) had psoriatic arthritis. JIA subsets did not statistically differ between patients with active disease and remission ( $p=0.388)$.

Serum lubricin levels did not differ between JIA patients (median: $0.66 \mathrm{ng} / \mu \mathrm{l}$, range: $0.02-3.85 \mathrm{ng} / \mu \mathrm{l}$ ) and healthy controls (median: $0.51 \mathrm{ng} / \mu \mathrm{l}$, range: $0.06-3.85 \mathrm{ng} / \mu \mathrm{l}$ ). However, it was statistically significantly higher in pa- 
Table II. Comparison of serum lubricin levels between juvenile idiopathic arthritis patients with active disease, remission and healthy controls

\begin{tabular}{|lcccc|}
\hline Parameter & $\begin{array}{c}\text { JIA with active disease } \\
(n=18)\end{array}$ & $\begin{array}{c}\text { JIA in remission } \\
(n=34)\end{array}$ & $\begin{array}{c}\text { Control group } \\
(n=28)\end{array}$ & $p$ \\
\hline Serum lubricin $(\mathrm{ng} / \mathrm{Ml})$, median (range) & $1.58(0.08-3.85)$ & $0.57(0.02-3.57)$ & $0.52(0.06-3.84)$ & 0.009 \\
\hline
\end{tabular}

$\| A$ - juvenile idiopathic arthritis, significant $p$-values $(<0.05)$ are given in bold.

tients with active disease than both patients in remission and healthy controls (Table II). Figure 1 demonstrates the comparison of the serum lubricin distribution between the groups. Unfortunately, we could not find any correlation between the number of active joints and the level of serum lubricin ( $r=-0.088, p=0.559)$.

We also compared current laboratory parameters and serum lubricin levels, and found a low degree positive correlation between serum lubricin levels and ESR $(r=0.383$ and $p=0.011)$ but not with $\operatorname{CRP}(r=0.265$, $p=0.086)$

\section{Discussion}

In our study, although serum lubricin levels were similar between overall JIA patients and controls, we found significantly elevated serum lubricin levels in active JIA patients, compared to the JIA patients in remission and healthy controls.

This is the first study investigating a possible anti-inflammatory role of lubricin in JIA patients. Although this is a preliminary study, in which lubricin was studied in only serum samples, we speculate that lubricin might be oversecreted from the synovial tissue in the inflammatory state of JIA. However, we did not identify any correlation between the number active joints and serum lubricin levels.

A complete lack of lubricin, resulting from homozygous PRG4 gene mutations, is the main cause of arthropathy in camptodactyly-arthropathy-coxa vara-pericarditis syndrome, which usually resembles JIA in childhood. Misdiagnosis of patients clinically, before diagnosis with genetic analysis, may be due to the fact that some patients also demonstrate inflammatory signs of arthritis [12, 13].

Previous studies investigated the pathogenetic roles of other proteins derived from synovial tissues in JIA patients. Kamphuis et al. [14] suggested that T-cells of JIA patients responded to self-epitopes derived from aggrecan, fibrillin, and matrix metalloproteinase 3. Degradation of aggrecan, a $P R G$ expressed mainly by chondrocytes, was also linked to the active phase of JIA in a previous study [15]. Another study showed increased plasma keratan sulphate levels in JIA patients, which decreased after treatment [16].

One-third of the dry weight of the cartilage matrix is formed by $P R G$ s, of which the majority is aggrecan,

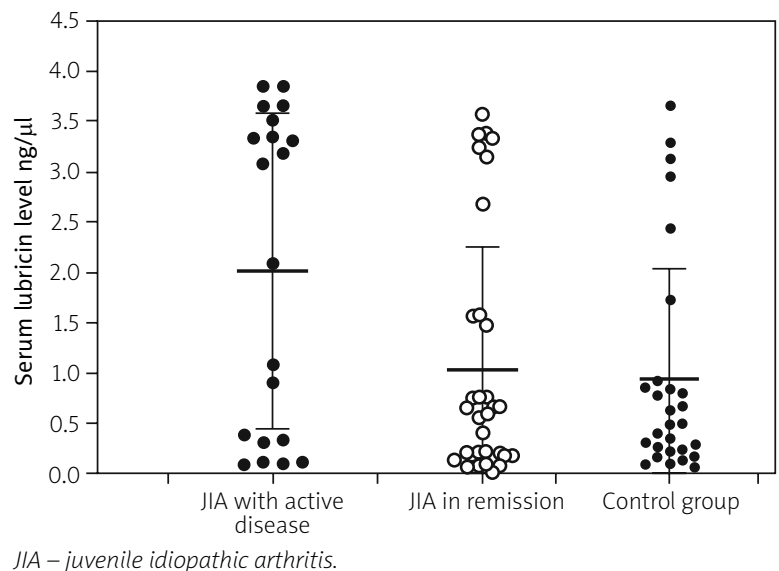

Fig. 1. Distribution of serum lubricin levels of juvenile idiopathic arthritis patients during active disease, remission and healthy controls

while others are decorin, biglycan, fibromodulin, lumican and PRG100 [17]. The strength of the cartilage was suggested to be determined by the aggregation of aggrecan with hyaluronic acid [17, 18]. The hyaluronic acid was elevated in systemic and polyarticular JiA in a former study [19]. Similarly, there are no other studies investigating the participation of alterations of other PRGs in JIA pathogenesis.

Moreover, lubricin was not also previously investigated in IIA patients, and not linked to a pathogenetic role different from other $P R G$ s, and we speculate that the involvement of lubricin in JIA pathogenesis may be overlooked so far.

Contrary to the lubricating function of lubricin, its role in inflammatory pathways could not be clarified yet. Former studies hypothesized that lubricin acts a buffer in the inflammatory response, via TLRs and CD44 binding $[6,7,20]$. However, a recent study suggested that lubricin plays a role in neutrophil recruitment to the inflamed joint in an L-selectin-dependent and -independent manner [21]. There is only one existing study which has investigated serum lubricin levels, instead of synovial fluid, in an inflammatory disease. In that study, serum lubricin levels were investigated among 30 rheumatoid arthritis (RA) patients, and no difference between serum lubricin levels of RA patients and control subjects was found. The authors reported a median serum lubricin level of 0.9 (range: 0.5-1.4 $\mathrm{ng} / \mu \mathrm{l}$ ), comparable to our 
study. The same study found a low-degree negative correlation between serum lubricin levels and CRP [22].

In contrast, our study showed elevated serum lubricin levels in active JA disease compared to JIA in remission and healthy controls. Although there was no correlation between serum lubricin levels and CRP, we found a low-degree positive correlation between ESR and serum lubricin levels. These conflicting results can only be clarified by further studies on this topic.

\section{Study limitations}

Our study has some limitations, including the lack of prospective design, limited number of different IA subtypes, and tissue levels of lubricin with inflammatory cytokines, particularly IL-1 $\beta$, IL- 6 and TNF- $\alpha$. From the results of our study, we cannot be sure if serum lubricin levels reflect the synovial fluid or tissue levels and interact with inflammatory cytokines. Moreover, all patients with active disease were under treatment, thus raising the question whether lubricin levels could be affected by antirheumatic medications. Nonetheless, we believe that this preliminary study will precede further work clarifying the role of lubricin in inflammatory conditions, particularly JIA.

These issues can be clarified with further longitudinal studies comparing lubricin along with inflammatory cytokines in the synovial biopsies of the same study samples in both active and treatment-naive and remission states.

\section{Conclusions}

This is the first study investigating serum lubricin levels in JIA patients, and we found elevated serum lubricin levels in JIA patients with active disease. Its exact role as an indicator of disease activity or response to treatment should be clarified by further comprehensive studies in the future.

\section{Acknowledgement}

We sincerely thank our mentor professor, Mustafa Yilmaz, for his great support in our pediatric rheumatology education. We are also grateful for his contribution to our professional life. We lost him two years ago, but he will be in our memories for the rest of our lives.

The authors declare no conflict of interest.

\section{References}

1. Schumacher BL, Block JA, Schmid TM, et al. A novel proteoglycan synthesized and secreted by chondrocytes of the superficial zone of articular cartilage. Arch Biochem Biophys 1994; 311: 144-152, DOI: 10.1006/abbi.1994.1219.
2. Schumacher BL, Schmidt TA, Voegtline MS, et al. Proteoglycan 4 (PRG4) synthesis and immunolocalization in bovine meniscus. J Orthop Res 2005; 23: 562-568, DOI: 10.1016/j. orthres.2004.11.011.

3. Flannery CR, Zollner R, Corcoran C, et al. Prevention of cartilage degeneration in a rat model of osteoarthritis by intraarticular treatment with recombinant lubricin. Arthritis Rheum 2009; 60: 840-847, DOI: 10.1002/art.24304.

4. Kosinska MK, Ludwig TE, Liebisch G, et al. Articular joint lubricants during osteoarthritis and rheumatoid arthritis display altered levels and molecular species. PLoS One 2015; 10: e0125192, DOI: 10.1371/journal.pone.0125192.

5. Estrella RP, Whitelock JM, Packer NH, Karlsson NG. The glycosylation of human synovial lubricin: implications for its role in inflammation. Biochem J 2010; 429: 359-367, DOI: 10.1042/ BJ20100360.

6. Alquraini A, Garguilo S, D'Souza G, et al. The interaction of lubricin/proteoglycan 4 (PRG4) with toll-like receptors 2 and 4: an anti-inflammatory role of $P R G 4$ in synovial fluid. Arthritis Res Ther 2015; 17: 353, DOI: 10.1186/s13075-015-0877-x.

7. Al-Sharif A, Jamal M, Zhang LX, et al. Lubricin/proteoglycan 4 binding to $\mathrm{cd} 44$ receptor: a mechanism of the suppression of proinflammatory cytokine-induced synoviocyte proliferation by lubricin. Arthritis Rheumatol 2015; 67: 1503-1513, DOI: 10.1002/art.39087.

8. Barut K, Adrovic A, Şahin S, Kasapçopur Ö. Juvenile idiopathic arthritis. Balkan Med J 2017; 34: 90-101, DOI: 10.4274/balkanmedj.2017.0111.

9. Prakken B, Albani S, Martini A. Juvenile idiopathic arthritis. Lancet 2011; 377: 2138-2149, DOI: 10.1016/S01406736(11)60244-4.

10. Petty RE, Southwood TR, Manners P, et al. International League of Associations for Rheumatology classification of juvenile idiopathic arthritis: second revision, Edmonton, 2001. J Rheumatol 2004; 31: 390-392.

11. Wallace CA, Giannini EH, Huang B, et al. American College of Rheumatology provisional criteria for defining clinical inactive disease in select categories of juvenile idiopathic arthritis. Arthritis Care Res (Hoboken) 2011; 63: 929-936, DOI: 10.1002/ acr.20497.

12. Kisla Ekinci RM, Balci S, Dogan H, et al. Camptodactyly-Arthropathy-Coxa Vara-Pericarditis Syndrome resembling juvenile idiopathic arthritis: a single-center experience from southern Turkey. Mol Syndromol 2021; 12: 112-117, DOI: 10.1159/000513111.

13. Yilmaz S, Uludağ Alkaya D, Kasapçopur Ö, et al. Genotype-phenotype investigation of 35 patients from 11 unrelated families with Camptodactyly-Arthropathy-Coxa Vara-Pericarditis (CACP) Syndrome. Mol Genet Genomic Med 2018; 6: 230-248, DOI: 10.1002/mgg3.364.

14. Kamphuis S, Hrafnkelsdóttir K, Klein MR, et al. Novel self-epitopes derived from aggrecan, fibrillin, and matrix metalloproteinase-3 drive distinct autoreactive T-cell responses in juvenile idiopathic arthritis and in health. Arthritis Res Ther 2006; 8: R178, DOI: 10.1186/ar2088.

15. Winsz-Szczotka K, Kuźnik-Trocha K, Komosińska-Vassev K, et al. Laboratory indicators of aggrecan turnover in juvenile 
idiopathic arthritis. Dis Markers 2016; 2016: 7157169, DOI: 10.1155/2016/7157169.

16. Winsz-Szczotka K, Komosińska-Vassev K, Kuźnik-Trocha K, et al. Circulating keratan sulfate as a marker of metabolic changes of cartilage proteoglycan in juvenile idiopathic arthritis; influence of growth factors as well as proteolytic and prooxidative agents on aggrecan alterations. Clin Chem Lab Med 2015; 53: 291-297, DOI: 10.1515/cclm-2014-0441.

17. Wojdas M, Dąbkowska K, Winsz-Szczotka K. Alterations of extracellular matrix components in the course of juvenile idiopathic arthritis. Metabolites 2021; 11: 132, DOI: 10.3390/ metabo11030132.

18. Vynios DH. Metabolism of cartilage proteoglycans in health and disease. Biomed Res Int 2014; 2014: 452315, DOI: 10.1155/2014/452315.
19. Shigemori M, Takei S, Imanaka H, et al. Diagnostic significance of increased serum hyaluronic acid in juvenile rheumatoid arthritis. Pediatr Int 2002; 44: 394-399, DOI: 10.1046/j.1442200x.2002.01586.x.

20. Iqbal SM, Leonard C, Regmi SC, et al. Lubricin/Proteoglycan 4 binds to and regulates the activity of toll-like receptors in vitro. Sci Rep 2016; 6: 18910, DOI: 10.1038/srep18910.

21. Jin C, Ekwall AK, Bylund J, et al. Human synovial lubricin expresses sialyl Lewis $\mathrm{x}$ determinant and has L-selectin ligand activity. J Biol Chem 2012; 287: 35922-35933, DOI: 10.1074/ jbc.M112.363119.

22. Ai M, Cui Y, Sy M-S, et al. Anti-lubricin monoclonal antibodies created using lubricin-knockout mice immunodetect lubricin in several species and in patients with healthy and diseased joints. PLoS One 2015; 10: e0116237, DOI: 10.1371/journal. pone.0116237. 